Dorota Weclawski, M.A. (Magistra Artium) Heinrich-Heine-University in Duesseldorf, Doropol company in Germany E-mail: dorota.weclawski@gmx.de ORCID ID 0000-0003-1549-4691

\title{
EXPOSÉ FOR SUCHIN'S BOOK: FAIRY PIECES OR CHESS FOR 2- TO 5 -YEAR-OLD CHILDREN"
}

\begin{abstract}
Annotation: In this article, the topic: "The didactic-methodical teaching of chess lessons in early childhood preschool age of two to five years" presented and the mutual exchange and communication based on it is for intercultural learning of great importance and plays not only in Germany in the concept the chess common Europe but is well on the way to developing progressively throughout the world.

Key words: Chess lessons, chess, fairy tales, synapses, analysis, chessboard, didactics and methodology.

\section{EXPOSÉ FÜR SUCHIN'S BUCH: "MÄRCHENHAFTE FIGUREN ODER SCHACH FÜR 2- bis 5 -JÄHRIGE KINDER"}

\begin{abstract}
Annotation: In diesem Artikel wird das Thema: „Die didaktisch-methodische Vermittlung des Schachspielunterrichts im frühkindlichen Vorschulalter von zwei bis fünf Jahren" dargestellt und der gegenseitiger Austausch und daraufbasierende Kommunikation ist für das interkulturelle Lernen von großer Bedeutung und spielt nicht nur in Deutschland beim Konzept des schachlich gemeinsamen Europas eine große Rolle, sondern ist auf dem guten Wege sich fortschreitend in der ganzen Welt zu entwickeln.
\end{abstract}

Schlüsselwörter: Schachspielunterricht, Schachspiel, Märchen, Synapsen, Analyse, Schachbrett, Didaktik und Methodik.

In der Einleitung des vorliegenden Schachbuches wird das Thema: „Die didaktischmethodische Vermittlung des Schachspielunterrichts im frühkindlichen Vorschulalter von zwei bis fünf Jahren" dargestellt.

Die Schachstudie, welche in der Prozessanalyse die strukturierte Vorgehensweise des Schachbeibringens aus wissenschaftlicher Sicht auf spieleriche Art erläutert, setzt sich aus vier unterschiedlich langen Kapiteln zusammen. Jedes Kapitel wird zu Beginn mit einem Dialog in Märchenform aufgebaut, welcher das Heranführen des Kindes an das Schachspiel einleitet und zum Schluß durch weitere methodische Hiweise für die schachspielenden Lehrer, Erzieher und Eltern ergänzt.

Den wissenschaftlichen Text könnte man im deutschen Schulschachsystem zum Beispiel auf die Märchen der Brüder Grimm übertragen. Auch wenn Märchen für die Forschung vor allem als wissenschaftliche und ethnologische Projekte dienen und politisch motiviert sind, bleibt das Kind mit Hilfe der Mythologie im eigenen Universum, in dem seine individuelle Kreativität mitels der Sozial- und Arbeitsformen durch die Interaktion im Bezug zu anderen Kindern gefördert wird. Um sein "autonomes Lernen" zu unterstützen, sollen durch das "mehrkanalige Lernen" möglichst alle Sinne des Kleinkindes beeinflusst, sinnvoll beschäftigt und miteinander verknüpft werden. Dabei werden mittels der Synapsen, die beiden Gehirnhälften, sowohl die rechte, welche die Kreativität als auch die linke, welche das logische Denken steuert, miteinander verbunden. Dadurch wird eine multiple und aktive Verarbeitung des Lernstoffes ermöglicht, die mittels des emotionalen Zugangs und Bezugs zur Lebenswelt, die Könnesentwicklung und das praktische Handeln des Vorschulalterkindes fördert. Seine 
Fähigkeiten sollen dabei ausgebaut und seine Fertigkeiten individuell entwickelt werden. Um das Schachkind dabei zu unterstützen, sollte der Lehrer den handlungsorientierten Unterricht durch den Einsatz möglichst vieler folgenden Unterrichtsmethoden, wie z.B.: die audio-visuelle (AVM) und audiolingualle (ALM), die kommunikative (KD) und direkte Methode (DM), sowie die immer wichtiger werdende interkulturelle Methode (IKM) auf eine einfache Weise mittels Didaktik und Methodik vermitteln.

"Zu bedenken ist dabei auch: Was ist den Lernern bekannt? Was ist neu und muss entsprechend eingeführt werden? Wo erwarten Sie Schwierigkeiten? Wie können Sie sie lösen? "Ob der Schachinstruktor das wissenschaftliche Kunstspiel ableitend oder erklärend vom Einfachen zum Schwierigen oder vom Allgemeinen zum Spezifischen vermitellt, ist für die Kinder nicht so wichtig wie der Spaß an dem Spiel selbst. Dabei ist es von großer Bedeutung den Träumen, der Fantasie und der Kreativität, sowie der Inspiration des Kindes freien Lauf zu lassen, um dabei zusätzlich seine Motivation zu steigern. Da nur so wird die benötigte Energie dafür entwickelt, die den fortlaufenden Lernprozess in Gang setzten kann. Wie wir alle wissen: "Lernen wird, wer lernen will."

Um die theoretischen Erkenntnisse anhand der Praxis aufzuzeigen, kommen wir auf die Analyse der wissenschaftlichen Schachstudie von Suchin zu sprechen.

Analyse des Textes: Im ersten Kapitel mit dem Titel "Bleistift malt die Schachfiguren", werden alle Figuren sowohl die weißen als auch die schwarzen in Märchendialog eingebettet und als Kontrastfiguren den Kindern malerisch vorgestellt. Im Schlußteil des Kapitels wird in den methodischen Hinweisen empfohlen, dass die Kinder erstmalig die Form, Symbolik und die Farbe der Schachfiguren mittels der Bilder spielerisch-malerisch und auf eine haptische Weise visualisieren und erkunden sollen. Diese Aspekte mit Hilfe der Plastizität beizubringen, betonen den spielerischen Gedanken dem Kleinkind das "zauberhafte" wissenschaftliche Kunstspiel mit mehr Spaß beizubringen, welcher stärker die Entwicklungsförderung seines Konzentrationsvermögens beeinflusst. Im Allgemeinen Spiele erleichtern den Kindern Kontakte in der Klasse und reduzieren Angst und Hemmungen bei der Entwicklung ihrer Kommunikationsfähigkeit. Das Annähern an die einfache Darstellung der Schachfiguren wird schwieriger in dem man sie symbolisch, z.B.: mittels der Märchendarstellung auf die konkreten Personen überträgt, wie z.B.: die Dame auf die Großmutter oder den König auf den Großvater, sowie die anderen Figuren auf die Tiere, wie den Turm auf den Bären, Springer auf den Fuchs und den Läufer auf den Wolf, sowie die Bauern auf die Hasen. Die Bilder, welche im Kopf des Kindes erzeugt und mit vorhanden Vorstellungen verknüpft werden, bestätigen die vorhanden Stereotypen und lösen bei ihm entweder weitere Neugier oder Ablehnung aus. Durch die Wahrnehmung des bekannten Bildes über das Verfahren der Bedeutungserschließung weiterhin zum unbekannten Bild lernt das Kleinkind bei den komplexen Aufgaben leichter zu reagieren. Diese Bildfunktion als infantiles Medium fördert die Konzentration und erleichtert speziell das Lernen und Behalten. Weiterhin ausgebaut spiegelt ein Bild die Welt einer Kultur des Landes wider, das eine große Rolle beim Prozess des interkulturellen Lernens spielt.

Im zweiten Kapitel "Zauberhafte Zellen" werden die quadratischen Bausteine in der Fachsprache Schachfelder genannt, als zuerst nebeneinander und dann getrennte glückliche oder traurige Gesichter auf spielerisch-malerische Weise dargestellt. Die kleinen Geschichtssegmente werden in eine Rahmenerzählung als Dialogform eingebettet. Dazu werden die weißen und schwarzen Felder, erstmal eins gegen eins, dann zwei gegen zwei usw. gegeneinander ausgespielt. Weiterhin werden mittels des versteckten Ziels die guten und schlechten Schachfelder dazu benutzt um verstärkt die entgegensetzte und dynamische Kraft der Schafiguren als auch die Wirkung der Diagonalmacht darzustellen. Das Endziel ist alle 
Schachfelder $(8 \times 8=64)$ auf dem Schachbrett von Buchstaben A bis $\mathrm{H}$ und von der Zahlen 1 bis 8 $\mathrm{zu}$ erkunden. Anschließend wird das Zentrum als wichtiger Ort der Zusammenkunft auf dem Schachbrett erklärt, indem man die gegensetzlichen Felder als Partner darstellt.

Im Hauptteil der Arbeit im dritten Kapitel "Der weiße Turm" werden im Suchin's Schachbuch erstmal alle schon bekannten Figuren als eine Art Wiederholung- um das Vorwissen zu aktivieren- aufgelistet. Dabei werden die Türme mit der Hilfe der Dame zu einem "hohen Turmbau" aufgestellt. Hierbei wird erweiternd die Dynamik im Schach eingeführt, bei der der Schachbegriff der Bewegung, d.h., des Schachzuges immer größere Bedeutung gewinnt. Erstmal wird der Turmzug mit Hilfe der Pfeile in Bezug auf die geographischen Himmelsrichtungen auf den Diagrammen dargestellt. Hierzu werden in den methodischen Hinweisen die Wirkung der weißen und schwarzen Felder, Linien und Reihen während des Turmzuges besser erläutert. Schach ist kein statisches sondern, dynamisches Spiel, deswegen müssen sich die Figuren in diesem Fall die Türme bemühen ihre dynamische Kraft noch mehr zu stärken und sich dabei sowohl horizontal auf den Linien als auch vertikal auf den Reihen bewegen, um gleichzeitig indirekt den sogenannten diagonalen Vektorraum abzudecken.

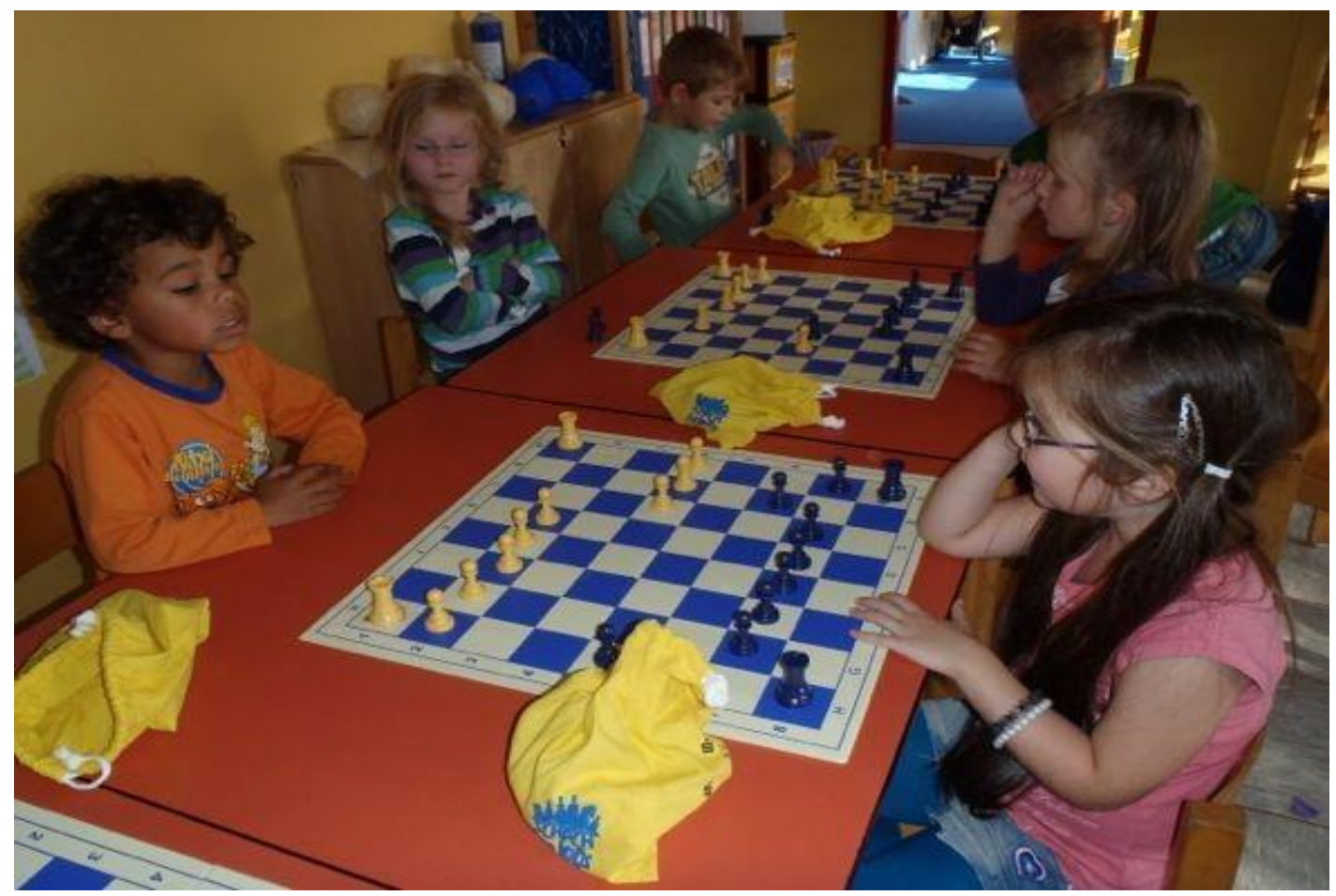

Kinder spielen Schach (Google search)

Im Abschlussteil der Schachstudie, das vierte Kapitel mit der shakespeareschen Allusion "Sein oder Nichtsein", weist starke fortlaufende Zusammenhänge zum dritten Kapitel auf. Die Lösungsregel der Aufgaben vom Einfachen zum Schweren, wird dabei sehr gut beachtet und eingesetzt. Hier wird die Bedeutung des Turmzuges erweiternd in Verbindung zu anderen Figuren gegenübergestellt. Dies wird noch übersichtlicher, wenn andere Schachfiguren, in dem Fall die anderen Türme ins Spiel kommen. Dadurch wird der Schachbegriff der Reihen näher erläutert, bei denen die Türme dem Tausch ausgeliefert oder der Schlaggefahr nicht ausgeliefert sind. Um dabei das ganze Schachbrett zu berücksichtigen, wird auch indirekt auf die die Wichtigkeit der Diagonalen hingewiesen. In diesem Kapitel wird die Bedeutung der statischen 
Schachfelder mit der dynamischen Bewegung der Schachfiguren in Verbindung gebracht. Um diese ausführlich zu üben, können die Stellungen der Schachfiguren auf den verschiedenen Feldern stark variiert werden. Dadurch wird die Absicht erreicht dem Kind das Alphabet und sogar die Zahlen, schachlich betrachtet die Buchstaben von A bis $\mathrm{H}$ und die Zahlen von 1 bis 8 , leichter beizubringen und vielleicht auf die Schachnotation hinzuweisen, damit die Kinder später die Schachpartien der großen Meister lesen und nachspielen können. Der weiße Turm kann sich dabei frei bewegen z.B.: vertikal auf der a-Reihe Ta1, dann Ta2 oder Ta3 oder horizontal auf der ersten Linie z.B., der Ta1 auf Tb1 oder weiter auf Tc1 usw. Und ob der Schachzug, die die Kinder gemacht haben, eher gut oder schlecht ist? Dazu werden erweitete schachlichen Symbole verwendet z.B. beim schlechten Zug das Symbol? beim sehr schlechten Zug ?? aber dafür beim guten Zug! und beim sehr guten!!. Außerdem gibt es auch Zeichen für ein interssante Idee !? oder doch nicht so interssanten Zug ?!, sowie beim Tausch oder Schlagen der Figuren ein Kreuz oder ein Doppelpunkt. Bei der ausführlichen Darstellung der Schachnotation werden alle Figuren und Schachfelder mittels verschiedener Züge schriftlich verfestigt. Daraus entwickelt sich ein Netzwerksystem, das die drei anderen Kapiteln der vorliegenden Schachstudie aufrundend zusammenfasst. Dabei werden die Sozial- und Kommunikationsformen mittels verschiedener Systeme den Kleinkindern vermittelt.

Schlußteil: Erwachsene haben im Gegensatz zu Kindern eine größere Lernerfahrung, da sie eher kognitiv den Lernstoff durch das Gliedern und Strukturieren verarbeiten und es besser auf das bereits Gelernte übertragen. Natürlich wird dem (Schach) Talent bei Kindern große Bedeutung beigemessen, da sie aber im Gegensatz zu Erwachsenen, durch die Imitation und somit mechanisch lernen, steht die Leistung nur im Hintergrund und wird auf spielerischeweise mit Hilfe von Motivation und Fleiß ausgetrickst, um trotzdem den Kurs mit dem Ziel der immer weiter entwickelnden Lernerfahrung im fortlaufendem Lernprozess zu steuern. Hier ist nicht wie bei den Erwachsenen die erhöhte Konzentrationsfähigkeit und das Durchhaltevermögen direkt so wichtig, sondern der Spaß an dem Spiel selbst, der auf die indirekteweise trotzdem dahinführt. Also wundern Sie sich nicht, wenn bald Ihr Schüler oder Kind, Ihnen schachlich zeigt, "wo der Hase hinläuft."

Um vielleicht nicht nur von der individuellen Entwicklungsmöglichkeiten zu sprechen, stellen wir ein anderes Ziel für die Kinder im Vorschulalter vor, die die Zukunft unserer Schulen und unseres Landes sind, um sie mittels der Interaktion mit anderen Sozialindividuen zu teilen.

Dazu genau passt das eher versteckte Ziel in der Suchin's Schachstudie, das sich in dem Konzept des Heranführens an das Schachspiel, die Absicht des Beibringens in Bezug auf das "interkulturelle Lernen" hinter versteckt. Der Schachtext beabsichtigt die Perspektive des „westlichen“ mit der des "östlichen" Denkens zu ergänzen. Durch das kulturelle Gesellschaftsspiel wird Schach nicht nur in der Freizeit, sondern auch in der Schule zum Näherbringen der Nationalitäten eingeleitet und darausresultiered die Neugierde des Kleinkindes nicht nur auf das Interesse der eigenen Kultur, sondern weiterhinaus dieses für andere Kulturen zu wecken. Dieser gegenseitiger Austausch und daraufbasierende Kommunikation ist für das interkulturelle Lernen von großer Bedeutung und spielt nicht nur in Deutschland beim Konzept des schachlich gemeinsamen Europas eine große Rolle, sondern ist auf dem guten Wege sich fortschreitend in der ganzen Welt zu entwickeln.

\section{Literaturquelle}

Сухин И. Г. Волшебные фигуры или шахматы для детей 2-5 лет. Москва, 1994 г. С. 1-28 / Suchin I. G. Märchenhafte Figuren oder Schach für Kinder von 2-5 Jahren. Moskau, 1994, S. 1-28. (In Russian) 